DOI: adma.201605123

Article type: Communication

\title{
The Formation of Defect-pairs for Highly Efficient Visible Light Catalysts
}

Qingbo Sun, David Cortie, Shaoyang Zhang, Terry J. Frankcombe, Guangwei She, Jie Gao, Leigh R. Sheppard, Wanbiao Hu, Hua Chen, Shangjun Zhuo, Dehong Chen, Ray L. Withers, Garry McIntyre, Dehong Yu, Wensheng Shi** and Yun Liu*

Mr. Q. Sun, Dr. D. Cortie, Dr. W. Hu, Dr. D. Chen, Prof. R. L. Withers, Prof. Y. Liu Research School of Chemistry, The Australian National University

Canberra, ACT 2601, Australia

E-mail: Yun.liu@anu.edu.au

Dr. D. Cortie, Dr. D. Yu, Prof. G. McIntyre

The Australian Nuclear Science and Technology Organisation

Lucas Height, NSW 2234, Australia

Mr. S. Zhang, Dr. G. She, Prof. W. Shi

Technical Institute of Physics and Chemistry, Chinese Academy of Sciences

Beijing 100190, China

E-mail:shiws@mail.ipc.ac.cn

Dr. T. J. Frankcombe

School of Physical, Environmental and Mathematical Sciences

The University of New South Wales

Canberra, ACT 2601, Australia

Mrs. J. Gao, Prof. S. Zhuo

Analysis and Testing Center for Inorganic Materials, Shanghai Institute of Ceramics

Chinese Academy of Sciences, Shanghai 200050, China

Dr. L. R. Sheppard

School of Computing, Engineering and Mathematics, Western Sydney University

Penrith, NSW 2751, Australia

Dr. H. Chen

Centre for Advanced Microscopy, The Australian National University

Canberra, ACT 2601, Australia

Keywords: defect-pairs, visible light, photocatalysts, titanium dioxide, solvothermal reaction

Abstract. Defect chemistry is widely used to modify materials for achieving high photocatalytic efficiency over a broad, in particular visible, light absorption range. Although various doping elements and associated effects have been extensively investigated, the lack of a fundamental understanding of the role of doping ions and a well-controlled synthetic strategy has hindered rational defect design in promising material systems, significantly impairing the development of highly efficient visible light catalysts (VLCs). In this work, we propose that fine-tuning the chemical scheme consisting of charge-compensated defect-pairs in balanced 


\section{WILEY-VCH}

concentrations is a key missing step. An experimental synthesis method was developed to form these defect-pairs in anatase $\mathrm{TiO}_{2}$ nanocrystals, resulting in a material with unprecedented VLC performance. Experimental and theoretical investigations indicate that the relatively highly concentrated $\mathrm{N}^{3-}-\mathrm{Nb}^{5+}$ defect-pairs enhance visible light absorption, prevent photo-generated charge carrier recombination, and significantly improve photocatalytic efficiency. We believe that this work not only unveils highly efficient $\mathrm{TiO}_{2}$-based VLCs for various photocatalytic applications but also provides general insight into the key importance of fine-tuning defect chemistry for functionalizing materials.

The gradual exhaustion of non-renewable resources has motivated researchers to investigate a wide range of materials for the highly efficient utilization of clean and renewable solar energy. Among these materials, visible light catalysts (VLCs) are vital and ideal for photo-degradation, dye-sensitized solar cells, hydrogen generation from water as well as organic synthesis with the potential to convert more than $\sim 45 \%$ of sunlight to chemical or electrical energy. Most of the current VLCs like $\mathrm{Ag}_{3} \mathrm{PO}_{4},{ }^{[1]} \mathrm{BiVO}_{4}{ }^{[2]}$ or $\mathrm{N}$ doped $\mathrm{TiO}_{2}{ }^{[3]}$ are only active in a certain solar spectral region and display limited photocatalytic performance. Other VLCs such as noble metal ions doped $\mathrm{SrTiO}_{3}{ }^{[4]}$ or decorated $\mathrm{TiO}_{2}{ }^{[5,6]}$ can extend the absorption edge to the whole visible light regime but they suffer from rapid photo-generated charge carrier recombination due to the presence of trapping centers (e.g. oxygen vacancies) or impurity energy levels. The recombination of electron-hole pairs not only suppresses the solar energy conversion efficiency but also deteriorates the photocatalytic activity of VLCs. Therefore, high performance photocatalysts should have broad light absorption while maintaining effective separation of electron-hole pairs in order to ensure the efficient utilization of solar energy for photocatalytic applications.

Technologically, several approaches have been proposed to date to enhance the photocatalytic performance of wide-band-gap semiconductors under visible light irradiation. For instance, the 


\section{WILEY-VCH}

lattice-disorder engineering of nanocrystalline $\mathrm{TiO}_{2}$ (via hydrogenation) enables an effective combination of visible and infrared absorption with the additional benefit of carrier trapping on surfaces. ${ }^{[7]}$ The design of a bandgap gradient distribution enables the solubility limits of undissolved ions to be increased and significantly inhibits the formation of recombination centers. ${ }^{[8]}$ Three-dimensionally-ordered construction of macroporous skeletons of N-doped $\mathrm{TiO}_{2}$ also greatly enhances visible-light driven photocatalytic properties by physically increasing surface area. ${ }^{[9]}$ These different approaches represent substantial progress in the research and development of novel VLCs.

Ionic co-doping, especially cation and anion co-doping, is another facile route. Co-doping has recently drawn considerable attention ${ }^{[10-20]}$ as this method can potentially tailor the energy band structure, enhance optical absorption, reduce the number of recombination centers by balancing charges and thus provide a cost-effective way to promote photocatalytic activity. The reported results, on the other hand, have been very diverse. There is no consensus concerning which type and what form of co-dopants should be introduced into host materials for achieving peak performance. Taking $\left(\mathrm{N}^{3-}, M^{5+}\right)$ co-doped $\mathrm{TiO}_{2}$ as an example, where $M^{5+}$ represents a pentavalent cation and $\mathrm{N}^{3-}$ is a nitrogen anion, it has been technologically difficult to introduce equivalent concentrations of $\mathrm{M}^{5+}$ and $\mathrm{N}^{3-}$ ions into a host material. High temperature ammonolysis ${ }^{[10-15]}$ was attempted to incorporate nitrogen ions into pre-prepared $M^{5+}$ monodoped $\mathrm{TiO}_{2}$ but the achieved $\mathrm{N}^{3-}$ doping level was less than 2 at.\%. ${ }^{[12,21]}$ In addition, the distribution of $\mathrm{N}^{3-}$ ions is often inhomogeneous with its concentration gradually lowering from the surface to the interior of co-doped $\mathrm{TiO}_{2} \cdot{ }^{[8]}$ Wet chemical methods like sol-gel synthesis have also been tried to directly add nitrogen sources to the reaction solution. ${ }^{[17-20]}$ However, the postcalcination treatment for crystallinity at temperatures over $400{ }^{\circ} \mathrm{C}$ inevitably leads to the loss of $\mathrm{N}^{3-}$ ions. ${ }^{[22]}$ Therefore, a well-balanced stoichiometric ratio of $\mathrm{M}^{5+}$ to $\mathrm{N}^{3-}$ has never been realized, although photocatalytic properties have been moderately improved. 


\section{WILEY-VCH}

From a theoretical perspective, there is also ambiguity concerning the electronic and chemical configurations of specific co-dopants in host materials and the related consequences for photocatalytic properties. The formation of impurity energy levels in the bandgap, ${ }^{[23]}$ the creation of mid-gap energy levels, ${ }^{[11,17]}$ the appearance of $\mathrm{Ti}^{3+}$ states and $\mathrm{F}^{+}$centers, ${ }^{[10,17,24]}$ interstitial nitrogen states ${ }^{[25]}$ as well as the red shift of the absorption edge ${ }^{[10,26]}$ have all been suggested as mechanisms for the observed enhancement of photocatalytic activity. Some investigators believe that cation-anion co-dopants can bind together to form defect-pairs ${ }^{[27,28]}$ while other work emphasizes the role of decoupled point defects formed under typical chemical conditions. ${ }^{[11,16]}$ Our recent work on the development of co-doped $\mathrm{TiO}_{2}$-based colossal permittivity materials ${ }^{[29,30]}$ demonstrated the complexity of ionic co-doping in structurally strongly correlated oxides. Once co-doped ions are introduced into host materials, they may stay as isolated point defects by either replacing ions in regular lattice sites or occupying interstitial positions. They may also form defect-pairs or clusters coupled with the host ions and local crystal structure. The former presents an additive effect of point defects and the latter results in a significant emergent behavior due to the combination of co-doped ions into a unique defect-pair or cluster form. The formation of defect states strongly depends on the preparation conditions and methods of material synthesis.

With this in mind, we have strategically designed a new approach for the development of $\left(\mathrm{N}^{3-}\right.$, $M^{5+}$ ) co-doped $\mathrm{TiO}_{2}$ with higher $\mathrm{N}^{3-}$ concentrations for a real synergistic and cooperative interaction between co-dopants. That is, another ion should be introduced to improve the local crystal chemical environment for accommodating more nitrogen ions. In this scenario, the two co-dopants must interact closely with each other within a small vicinity to form defect-pairs or clusters. The impact of newly formed defect-pairs or clusters would be expected to behave as a new type of defect which not only has the special features of individual dopant but also effectively avoids the drawbacks of single doping. In the case of $\left(\mathrm{N}^{3-}, \mathrm{Nb}^{5+}\right)$ co-doped anatase 


\section{WILEY-VCH}

$\mathrm{TiO}_{2}$ nanocrystals, the following factors were further considered: (1) the well-balanced charges of $\mathrm{N}^{3-}$ and $\mathrm{Nb}^{5+}$ ions to the maximum extent avoiding the generation of additional defects such as $\mathrm{Ti}^{3+}$ ions or oxygen vacancies $\left(V_{O}^{\bullet \bullet}\right)$; (2) the more difficult reduction of $\mathrm{Nb}^{5+}$ ions than $\mathrm{Ti}^{4+}$ ions to keep the chemical valence of niobium dopants experimentally controllable; and (3) the formation of strong correlation (defect-pairs) between $\mathrm{N}^{3-}$ and $\mathrm{Nb}^{5+}$ ions to activate significant emergent behaviors for the physicochemical properties. The resultant materials were subsequently characterized as VLCs for water purification using Rhodamine B as a model pollutant. Density functional theory (DFT) calculations were performed to gain insight into the observed photocatalytic effects and the related mechanisms arising from the formation of highly concentrated $\mathrm{N}^{3-}-\mathrm{Nb}^{5+}$ defect-pairs. This study demonstrates how to design and develop novel photocatalytic materials for highly efficient conversion of solar energy.

In order to efficiently introduce nitrogen ions into host materials and simultaneously avoid nitrogen evaporation during subsequent high temperature processing, a solvothermal reaction route was used in this work for material synthesis. This enables the chemical reaction to occur homogenously at the molecular level under pressure and at a lower temperature. This method also prevents the loss of nitrogen ions during reaction due to the closed environment. For instance, to successfully synthesize $(\mathrm{N}, \mathrm{Nb})$ co-doped $\mathrm{TiO}_{2}, \mathrm{NbCl}_{5}$ and $\mathrm{TiCl}_{4}$ are dissolved in ethanol and chemically reacted with nitric acid at $200{ }^{\circ} \mathrm{C}$ for 12 hours. ${ }^{[31]}$ Repeating this reaction procedure, we synthesized a series of $(\mathrm{N}, \mathrm{Nb})$ co-doped $\mathrm{TiO}_{2}$ samples, where the nominal concentration of $\mathrm{Nb}^{5+}$ ions was fixed during the whole experimental process while the nominal $\mathrm{N}^{3-}$ doping level was varied by adjusting the concentration of nitric acid in the reaction solution (Supporting Information, SI-1). After optimization, the structure and chemical compositions of the resultant solvothermal products were determined by X-ray powder diffraction (XRPD), high-resolution transmission electron microscopy (HRTEM), field-emission scanning electron

microscope (FESEM) and X-ray photoelectron spectroscopy (XPS). Additionally, a 


\section{WILEY-VCH}

Nitrogen/Oxygen determinator, thermogravimetric analysis (TGA) and differential scanning calorimetry (DSC) were also used to further estimate the resultant nitrogen concentrations (SI6), which were found to be reasonably consistent with the results determined by XPS. The experimental details are presented in SI-2.

The typically synthesized samples were experimentally analyzed to have $\mathrm{Nb}^{5+}$ ionic concentrations of about 5.4, 5.3 and 5.6 at.\% per $\left(\mathrm{Ti}_{1-\mathrm{x}-\mathrm{y}}^{4+} \mathrm{Ti}_{\mathrm{x}}^{3+} \mathrm{Nb}_{\mathrm{y}}^{5+}\right)\left(\mathrm{O}_{2-\mathrm{y}{ }^{\prime}-\mathrm{z}}^{2-} \square_{z} \mathrm{~N}_{\mathrm{y}}^{3-}\right)$ formula unit and $\mathrm{N}^{3-}$ ionic concentrations of about 3.7, 5.6 and 7.5 at.\% (labelled as N-poor, N-equal and Nrich), respectively (SI-5 and 6). XRPD analysis (Figure 1a) suggests that all prepared (N, Nb) co-doped $\mathrm{TiO}_{2}$ particles belong to the anatase phase with space group symmetry $\mathrm{I}_{1} /$ amd. The diffraction peaks are very broad, indicating that the materials have a small particle size, further calculated via the Scherrer equation (Equation S1) to show their average diameters are less than $10 \mathrm{~nm}$ (SI-8). This is also confirmed by a FESEM image (SI-7a), but more accurately estimated by HRTEM lattice images (Figure 1c, SI-7 and 8). From $<100>$ oriented HRTEM lattice images (Figure 1c and the inserted images of SI-7c and d), it is found that the dominant exposed crystal faces of these three different $(\mathrm{N}, \mathrm{Nb})$ co-doped $\mathrm{TiO}_{2}$ nanoparticles are $\{011\}$ facets combined, to a lesser extent, with (002) facets. Furthermore, the three types of samples have almost equivalent specific surface area $\left(\sim 170 \mathrm{~m}^{2} / \mathrm{g}\right.$, SI-9). In addition, we observed that the color of the synthesized nanoparticles varied from light brown to dark brown with increasing $\mathrm{N}^{3-}$ concentrations (Figure 1b). This color variation clearly indicates a change in their visible light absorption, as will be further demonstrated below. From XPS analysis (Figure 1d-f and SI-5), it is found that some $\mathrm{Ti}^{4+}$ ions of the $\mathrm{N}$-poor $\mathrm{TiO}_{2}$ samples are reduced to $\mathrm{Ti}^{3+}$ ions to compensate for the additional positive charges associated with the excessive $\mathrm{Nb}^{5+}$ ions. As a result of charge compensation, the total doping concentrations of $\mathrm{Ti}^{3+}\left(1.7\right.$ at.\%) and $\mathrm{N}^{3-}(3.7$ at.\%) are equal to that of $\mathrm{Nb}^{5+}$ (5.4 at.\%). Similarly, oxygen vacancies ( $V_{O}^{\bullet \bullet}$, labelled as $\square_{\mathrm{z}}$ in the formula unit above) are generated in the $\mathrm{N}$-rich co-doped $\mathrm{TiO}_{2}$ to balance the extra negative 


\section{WILEY-VCH}

charges arising from the excess $\mathrm{N}^{3-}$ ions. If $\mathrm{Nb}^{5+}$ and $\mathrm{N}^{3-}$ ions are doped at equal concentrations i.e. if $\mathrm{y}=\mathrm{y}^{\prime}$, neither $\mathrm{Ti}^{3+}$ ions nor $V_{O}^{\bullet \bullet}$ defects would be generated. In fact, no such defects are observed even in our $\mathrm{N}$-equal co-doped samples where the concentrations of $\mathrm{Nb}^{5+}$ and $\mathrm{N}^{3-}$ are not exactly identical but very close (5.3 at. $\% \mathrm{Nb}^{5+}+5.6$ at.\% $\left.\mathrm{N}^{3-}\right)$. It is evident that the $\mathrm{N}$ doping level per formula unit can reach up to 7.5 at.\% with $\mathrm{Nb}^{5+}$ co-doping using this solvothermal reaction route. Such a high $\mathrm{N}^{3-}$ doping level suggests that $\mathrm{Nb}^{5+}$ co-doping facilitates the introduction of $\mathrm{N}^{3-}$ ions into anatase $\mathrm{TiO}_{2}$.

Figure 2a shows the optical absorption spectra of (N, Nb) co-doped $\mathrm{TiO}_{2}$ nanoparticles. Their light absorption range covers the entire visible light regime from $400 \mathrm{~nm}$ to $800 \mathrm{~nm}$, which is much broader, relative to the results previously reported for $(\mathrm{N}, \mathrm{Nb})$ co-doped $\mathrm{TiO}_{2}$ particles. ${ }^{[10-}$ ${ }^{15,17-20]}$ For the $\mathrm{N}$-equal and $\mathrm{N}$-rich co-doped $\mathrm{TiO}_{2}$ with higher $\mathrm{N}^{3-}$ doping levels, the respective light absorption even extends into the near infrared regime. The strongest light absorption is observed in our $\mathrm{N}$-equal $\mathrm{TiO}_{2}$ with almost identical $\mathrm{Nb}^{5+}$ and $\mathrm{N}^{3-}$ doping concentrations (5.3 at.\% $\mathrm{Nb}^{5+}+5.6$ at. $\% \mathrm{~N}^{3-}$ ). This is in good agreement with their color change (Figure $1 \mathrm{~b}$ ). In addition, the Tauc plots of Figure $2 \mathrm{~b}$ show that the bandgap of these co-doped $\mathrm{TiO}_{2}$ materials becomes significantly narrower with increasing $\mathrm{N}^{3-}$. The bandgap values are $\sim 2.9 \mathrm{eV}$ and $\sim 2.6 \mathrm{eV}$ for $\mathrm{N}$ poor and N-rich samples, respectively. The $\mathrm{N}$-equal $\mathrm{TiO}_{2}$ is even better with a bandgap reduction to $\sim 2.2 \mathrm{eV}$, demonstrating the importance of fine-tuning chemical scheme to give charge-compensated defects in balanced concentrations.

The photocatalytic activity of these VLCs is investigated by measuring the decomposition of Rhodamine B (RhB) under visible light irradiation, using a 500W Xe lamp with a cutoff wavelength of $400 \mathrm{~nm}$. Experimental details are presented in SI-3. Compared with the commercial Degussa $\mathrm{P} 25-\mathrm{TiO}_{2}$, the photocatalytic activity of all three (N, Nb) co-doped anatase $\mathrm{TiO}_{2}$ samples is superior and the $\mathrm{N}$-equal co-doped $\mathrm{TiO}_{2}$ is outstanding (Figure 2c). As seen, 


\section{WILEY-VCH}

the $\mathrm{N}$-equal co-doped $\mathrm{TiO}_{2}$ requires less than 20 minutes to accomplish nearly complete decomposition of RhB. It is noticed that the N-poor, N-equal and N-rich co-doped $\mathrm{TiO}_{2}$ nanocrystals have similar average particle sizes, the analogous crystal facets exposed on the surface, almost equivalent specific surface area (SI-9), but show a clear difference in VLC effect. Hence, it is concluded that $\mathrm{N}^{3-}$ and $\mathrm{Nb}^{5+}$ ionic co-doping, which has been experimentally realized by the solvothermal reaction route, is critical to stabilize the $\mathrm{N}$-doping and/or accommodate more $\mathrm{N}^{3-}$ ions in the host $\mathrm{TiO}_{2}$. High co-dopant loading is significant to improve photocatalytic activity, but the charge-compensated defects in balanced $\mathrm{Nb}^{5+}$ and $\mathrm{N}^{3-}$ concentrations are a decisive factor influencing the outstanding visible-light-catalytic performance in this material.

To elucidate how the $(\mathrm{N}, \mathrm{Nb})$ co-doping strategy modifies the electronic structure and leads to the enhanced VLC performance, first principles calculations were performed. Figure 3a-c shows some of the candidate chemical structures that were modelled using DFT calculations for the $(\mathrm{N}, \mathrm{Nb})$ co-doped $\mathrm{TiO}_{2}$ compound. The starting configurations were generated by substituting equivalent $\mathrm{Nb}$ and $\mathrm{N}$ into various Ti and $\mathrm{O}$ sites with dopant concentrations ( 6.25 at.\%) close to the optimal experimental values ( 5.3 at.\% $\mathrm{Nb}^{5+}+5.6$ at.\% $\left.\mathrm{N}^{3-}\right)$, and then allowing the ions to relax to low energy configurations using the Perdew-Burke-Ernzerhof (PBE) functional. Table 1 reports the total energies, indicating that it is energetically favorable to position substitutional $\mathrm{N}-\mathrm{Nb}$ in a nearest neighbor configuration (Structure I). Indeed, the energy cost for positioning N-Nb further apart is $\sim 0.1 \mathrm{eV}$ (Structure II and III), setting the corresponding temperature scale in the thermodynamic limit as $\Delta E / k_{B}=1160 \mathrm{~K}$ where $k_{B}$ is the Boltzmann constant. For this reason, the formation of structures such as II and III are improbable under the experimental synthesis conditions of this work and the local crystal chemistry favors the formation of directly-bonded N-Nb configurations, i.e. defect-pairs. 


\section{WILEY-VCH}

To model the electronic effects of the resultant defect-pairs, the density of states (DOS) was calculated using the modified-Becke-Johnson $(\mathrm{mBJ})$ potential which is a parameter-free ab initio method for the bandgap prediction. ${ }^{[32,33]}$ The total DOSs for un-doped and $(\mathrm{N}, \mathrm{Nb})$ codoped anatase $\mathrm{TiO}_{2}$ with various doped structures (I-III) are shown in Figure 3d. The calculated bandgap lies within $10 \%$ of the experimental values, for example, producing $2.98 \mathrm{eV}$ for undoped anatase $\mathrm{TiO}_{2}$ (experimental value 3.1-3.2 eV). ${ }^{[34]}$ All of the structures that contain $\mathrm{Nb}$ and $\mathrm{N}$ have a reduced bandgap compared to the pure anatase structure, with values close to 2.4 $\mathrm{eV}$ (Table 1). This corroborates the main optical bandgap ( $2.2 \mathrm{eV})$ observed in the experiment (Figure 2b). The first principles calculations hence again indicate some cooperative electronic effect of the $\mathrm{Nb}$ and $\mathrm{N}$ species. Figure 3e shows the element-specific partial DOSs for the nearest-neighbor $\mathrm{N}-\mathrm{Nb}$ structure I demonstrating that the $\mathrm{Nb}$ levels are high in the conduction band whereas the $\mathrm{N}$ forms a shallow acceptor level that almost overlaps the valence band maxima. The combination of these properties ensures thermal occupation under typical experimental conditions, providing an effective way to engineer the band-edge. Moreover, the Fermi energy offset from the valence band (taken as the $\mathrm{E}=0$ ) is not modified compared to pure anatase $\mathrm{TiO}_{2}$, illustrating the advantages of the passivated co-doping involved with the $\mathrm{N}^{3-}$ $\mathrm{Nb}^{5+}$ defect-pairs. This enhances photocatalytic performances by preventing unwanted recombination pathways for photo-generated charge carriers. ${ }^{[28]}$

To directly show that the $\mathrm{N}^{3-}-\mathrm{Nb}^{5+}$ defect-pairs also enhance the harvesting of visible light, we calculated the optical absorption predicted for the resulting electronic structure. Figure 3f shows the absorption coefficient for the nearest-neighbor $\mathrm{N}^{3-}-\mathrm{Nb}^{5+}$ defect-pair structure I based on the joint-density-of-states method. ${ }^{[35]}$ Both absorption tensor components ( $x x$ and $z z$ ) indicate finite absorption into the visible regions, tailing off rapidly near the indirect bandgap energy (516 $\mathrm{nm})$. By contrast, we also report the electronic-structure calculations for interstitial nitrogen species (SI-10) as some researchers think that they might play a crucial role in nitrogen mono- 


\section{WILEY-VCH}

doped $\mathrm{TiO}_{2} \cdot{ }^{[36]}$ However, the calculated results for such interstitial nitrogen species in the neighborhood of $\mathrm{Nb}^{5+}$ ions show that light absorption is only achieved below $440 \mathrm{~nm}(\sim 2.82$ $\mathrm{eV}$ ), which represents a significant drop in light-harvesting performance. Clearly, the electronic structure originating from the $\mathrm{N}^{3-}-\mathrm{Nb}^{5+}$ defect-pairs in closely-neighboring substitutional positions is key to producing enhanced visible absorption which is necessary for high photocatalytic performance.

In summary, this work introduces the concept of defect-pairs to make anatase $\mathrm{TiO}_{2}$ a strikingly superior VLC. This is because the formation of highly concentrated defect-pairs, in this case $\mathrm{N}^{3-}-\mathrm{Nb}^{5+}$, will greatly reduce the bandgap, enhance visible light absorption and diminish photoexcited charge carrier recombination. The realization of high nitrogen concentrations benefits from the defect design and the formation of $\mathrm{N}^{3-}-\mathrm{Nb}^{5+}$ defect-pair structure. Solvethermal reaction provides an effective approach to further promote the achievement of such high level of co-dopants. Given that the improvements in the $\mathrm{TiO}_{2}$-based VLC performence have been very limited despite massive efforts over the past three decades, this work provides a breakthrough in the design of $\mathrm{TiO}_{2}$-based VLCs. The new mechanism and associated strategy we have developed here not only elevate $\mathrm{TiO}_{2}$ to the ranks of the best available VLCs, thus benefiting all practical applications, but also provide new insights to improve existing and newly developing VLCs for a clean environment (e.g. waste water/chemical treatment) and renewable energy (e.g. water splitting for hydrogen generation).

\section{Experimental Section}

The detailed information about the synthesis and analysis of $(\mathrm{N}, \mathrm{Nb})$ co-doped anatase $\mathrm{TiO}_{2}$ nanocrystals, the characterization of photocatalytic performance, density functional theory calculations and other related materials are available in the Supporting Information.

\section{Supporting Information}

Supporting Information (SI) is available from the Wiley Online Library or from the author.

\section{Acknowledgements}

QS, DCo, TJF and YL acknowledge the supports of the Australian Research Council (ARC) in the form of Discovery Projects. YL and TJF also appreciate the support from the ARC Future Fellowships program. SZ, GS and WS thank the financial support from CAS 


\section{WILEY-VCH}

(XDB17030000) and MOST of China (2016YFA0200800). The authors also appreciate Antonio Tricoli and Guanyu Liu (the Australian National Univerity) for their assistance in the B.E.T. measurement and Bill Gong (University of New South Wales) for his help in XPS characterization. The authors acknowledge the facilities and the scientific and technical assistance of the Australian microscopy and microanalysis research facility (AMMRF) at the Centre of advanced Microscopy, the Australian National University.

\section{Authors' contributions}

QS, DCo, YL, WS and TJF make the main contribution to the preparation of this manuscript. YL initiated this research, planned and coordinated all experimental and theoretical work. WS and TJF supervised the photocatalytic and theoretical work collaborated with YL. QS developed the approach for the fabrication of the samples with different compositions and synthetic conditions. SZ and GS conducted the photocatalytic experiment. DCo conducted theoretical calculation, collaborated with GM and DY. QS, WH, RLW, LS and DCh conducted HRTEM, SEM/EDS, XPS and light absorption with the assistance of HC and LS. JG and SZ conducted the chemical composition analysis relevant to nitrogen. All authors were involved in the data analysis and discussion as well as manuscript preparation.

\section{Competing financial interests}

The authors declare no competing financial interest.

\section{References}

[1] Z. Yi, J. Ye, N. Kikugawa, T. Kako, S. Ouyang, H. Stuart-Williams, H. Yang, J. Cao, W. Luo, Z. Li, Y. Liu, R. L. Withers, Nat. Mater. 2010, 9, 559.

[2] T. W. Kim, Y. Ping, G. A. Galli, K. -S. Choi, Nat. Commun. 2015, 6, 8769.

[3] R. Asahi, T. Morikawa, T. Ohwaki, K. Aoki, Y. Taga, Science 2001, 293, 269.

[4] K. Iwashina, A. Kudo, J. Am. Chem. Soc. 2011, 133, 13272.

[5] Q. Zhang, D. Q. Lima, I. Lee, F. Zaera, M. Chi, Y. Yin, Angew. Chem. 2011, 123, 7226.

[6] L. Jing, W. Zhou, G. Tian, H. Fu, Chem. Soc. Rev. 2013, 42, 9509.

[7] X. Chen, L. Liu, P. Y. Yu, S. S. Mao, Science 2011, 331, 746.

[8] G. Liu, L. -C. Yin, J. Wang, P. Niu, C. Zhen, Y. Xie, H. -M. Chen, Energy Environ. Sci. 2012, 5, 9603.

[9] T. Wang, X. Yan, S. Zhao, B. Lin, C. Xue, G. Yang, S. Ding, B. Yang, C. Ma, G. Yang, G. Yang, J. Mater. Chem. A 2014, 2, 15611.

[10] T. M. Breault, B. M. Bartlett, J. Phys. Chem. C 2012, 116, 5986. 


\section{WILEY-VCH}

[11] C. Marchiori, G. D. Liberto, G. Soliveri, L. Loconte, L. L. Presti, D. Meroni, M. Ceotto,

C. Oliva, S. Cappelli, G. Cappelletti, C. Aieta, S. Ardizzone, J. Phys. Chem. C 2014, 118, 24152.

[12] T. M. Breault, J. J. Brancho, P. Guo, B. M. Bartlett, Inorg. Chem. 2013, 52, 9363.

[13] S. Hoang, S. Guo, C. B. Mullins, J. Phys. Chem. C 2012, 116, 23283.

[14] T. Cottineau, N. Béalu, P. -A. Gross, S. N. Pronkin, N. Keller, E. R. Savinova, V. Keller, J. Mater. Chem. A 2013, 1, 2151.

[15] T. M. Breault, B. M. Bartlett, J. Phys. Chem. C 2013, 117, 8611.

[16] H. Choi, D. Shin, B. C. Yeo, T. Song, S. S. Han, N. Park, S. Kim, ACS Catal. 2016, 6, 2745.

[17] J. Lim, P. Murugan, N. Lakshminarasimhan, J. Y. Kim, J. S. Lee, S. -H. Lee, W. Choi, J. Catal. 2014, 310, 91.

[18] P. Zhang, S. Yin, T. Sekino, S. W. Lee, T. Sato, Res. Chem. Intermed. 2013, 39, 1509.

[19] Y. Shen, T. Xiong, J. Shang, K. Yang, Res. Chem. Intermed. 2008, 34, 353.

[20] L. Rimoldi, C. Ambrosi, G. D. Liberto, L. L. Presti, M. Ceotto, C. Oliva, D. Meroni, S. Cappelli, G. Cappelletti, G. Soliveri, S. Ardizzone, J. Phys. Chem. C 2015, 119, 24104.

[21] C. Burda, Y. Lou, X. Chen, A. C. S. Samia, J. Stout, J. L. Gole, Nano Lett. 2003, 3, 1049.

[22] T. C. Jagadale, S. P. Takale, R. S. Sonawane, H. M. Joshi, S. I. Patil, B. B. Kale, S. B. Ogale, J. Phys. Chem. C 2008, 112, 14595.

[23] Q. Meng, T. Wang, E. Liu, X. Ma, Q. Ge, J. Gong, Phys. Chem. Chem. Phys. 2013, 15, 9549.

[24] A. Folli, J. Z. Bloh, A. Lecaplain, R. Walker, D. E. Macphee, Phys. Chem. Chem. Phys. 2015, 17, 4849.

[25] S. Banerjee, S. C. Pillai, P. Falaras, K. E. O’Shea, J. A. Byrne, D. D. Dionysiou, J. Phys. Chem. Lett. 2014, 5, 2543. 


\section{WILEY-VCH}

[26] R. Long, N. J. English, Chem. Phys. Lett. 2009, 478, 175.

[27] X. Ma, Y. Wu, Y. Lu, J. Xu, Y. Wang, Y. Zhu, J. Phys. Chem. C 2011, 115, 16963.

[28] Y. Gai, J. Li, S. -S. Li, J. -B. Xia, S. -H. Wei, Phys. Rev. Lett. 2009, 102, 036402.

[29] W. Hu, Y. Liu, R. L. Withers, T. J. Frankcombe, L. Norén, A. Snashall, M. Kitchin, P.

Smith, B. Gong, H. Chen, J. Schiemer, F. Brink, J. Wong-Leung, Nat. Mater. 2013, 12, 821.

[30] W. Hu, K. Lau, Y. Liu, R. L. Withers, H. Chen, L. Fu, B. Gong, W. Hutchison, Chem.

Mater. 2015, 27, 4934.

[31] Y. Liu, Q. Sun, R. L. Withers, W. Shi, Au. 2016903458, 2016.

[32] F. Tran, P. Blaha, Phys. Rev. Lett. 2009, 102, 226401.

[33] P. Blaha, K. Schwarz, G. K. H. Madsen, D. Kvasnicka, J. Luitz, WIEN2k: an augmented plane wave + local orbitals program for calculating crystal properties. Techn. Universität, Wien, AT 2001.

[34] D. O. Scanlon, C. W. Dunnill, J. Buckeridge, S. A. Shevlin, A. J. Logsdail, S. M.

Woodley, C. R. A. Catlow, M. J. Powell, R. G. Palgrave, I. P. Parkin, G. W. Watson, T. W.

Keal, P. Sherwood, A. Walsh, A. A. Sokol, Nat. Mater. 2013, 12, 798.

[35] C. Ambrosch-Draxl, J. O. Sofo, Comput. Phys. Commun. 2006, 175, 1.

[36] M. Ceotto, L. L. Presti, G. Cappelletti, D. Meroni, F. Spadavecchia, R. Zecca, M. Leoni, P. Scardi, C. L. Bianchi, S. Ardizzone, J. Phys. Chem. C 2012, 116, 1764.

Received: ((will be filled in by the editorial staff))

Revised: ((will be filled in by the editorial staff)) Published online: ((will be filled in by the editorial staff)) 


\section{WILEY-VCH}
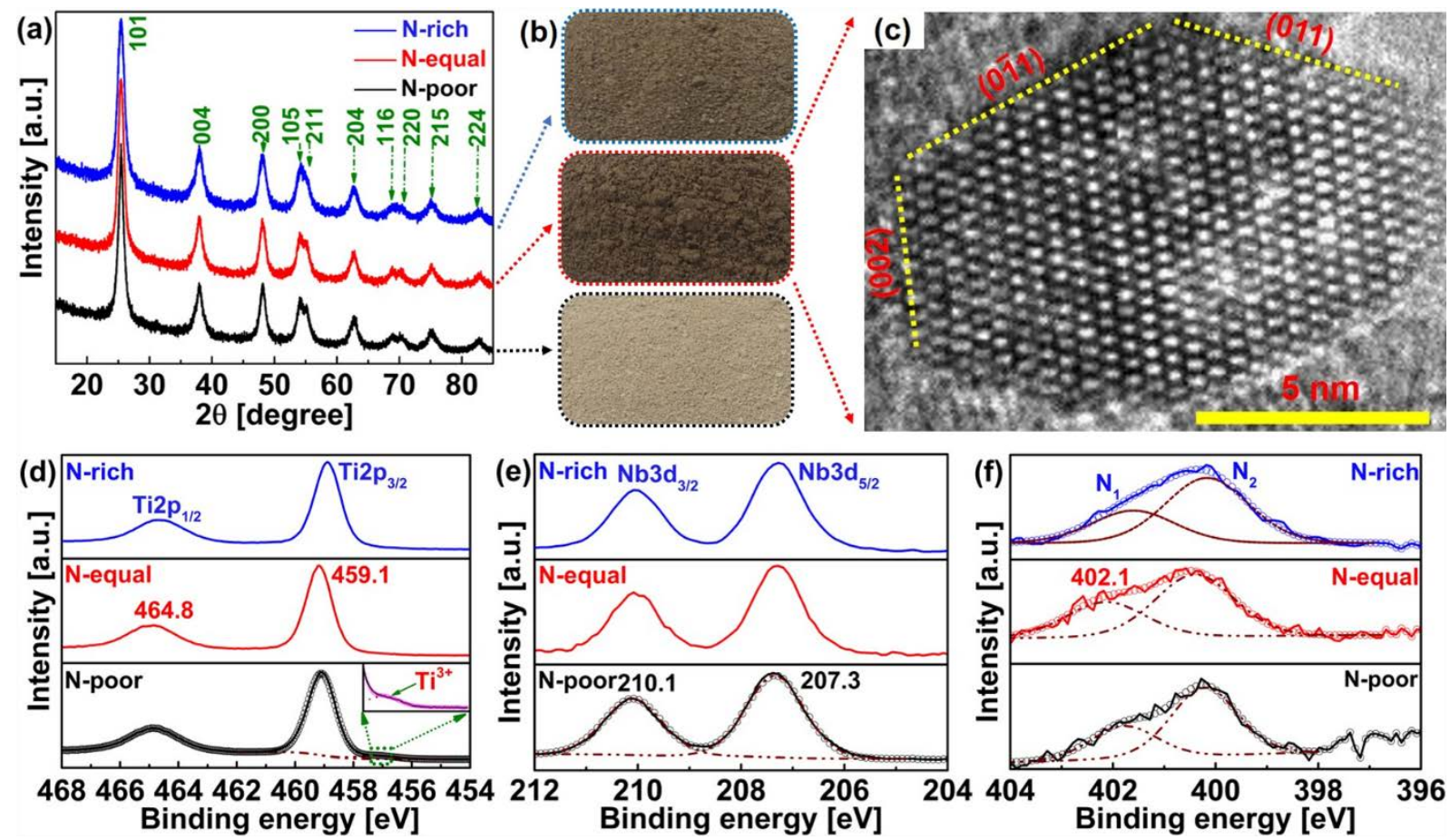

Figure 1. XRD patterns (a), photographs (b), a HRTEM lattice image along a $<100>$ direction (c) and XPS data (d-f) of $\mathrm{N}$-poor (5.4 at.\% $\mathrm{Nb}^{5+}+3.7$ at. $\% \mathrm{~N}^{3-}$ ), $\mathrm{N}$-equal (5.3 at.\% $\mathrm{Nb}^{5+}+5.6$ at.\% $\mathrm{N}^{3-}$ ) and $\mathrm{N}$-rich (5.6 at.\% $\mathrm{Nb}^{5+}+7.5$ at.\% $\mathrm{N}^{3-}$ ) co-doped $\mathrm{TiO}_{2}$ nanocrystals which were synthesized by the solvothermal reaction.
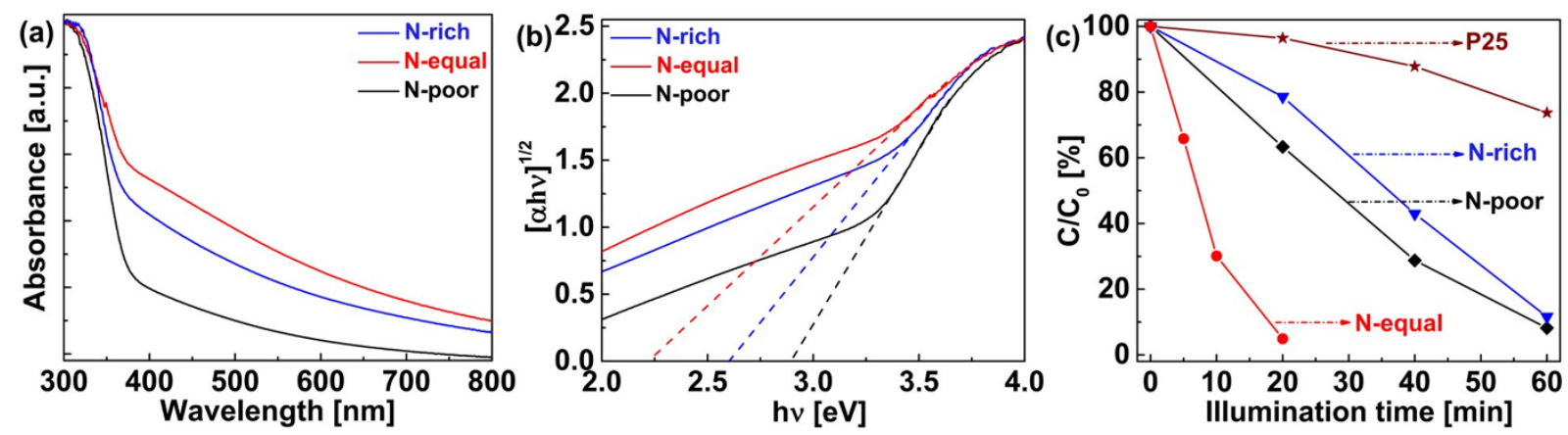

Figure 2. UV-Vis absorption spectra (a) and associated Tauc plots (b) of three typical N-poor (5.4 at.\% $\mathrm{Nb}^{5+}+3.7$ at.\% $\mathrm{N}^{3-}$ ), N-equal (5.3 at.\% $\mathrm{Nb}^{5+}+5.6$ at.\% $\mathrm{N}^{3-}$ ) and N-rich (5.6 at.\% $\mathrm{Nb}^{5+}+7.5$ at. $\% \mathrm{~N}^{3-}$ ) co-doped $\mathrm{TiO}_{2}$ nanocrystals. (c) presents the photocatalytic degradation of Rhodamine $B$ under visible light irradiation $(\lambda \geq 400 \mathrm{~nm})$ using these nanocrystals as visible light catalysts, where the commercial Degussa $\mathrm{P} 25-\mathrm{TiO}_{2}$ is also measured for a reference. 
(a)

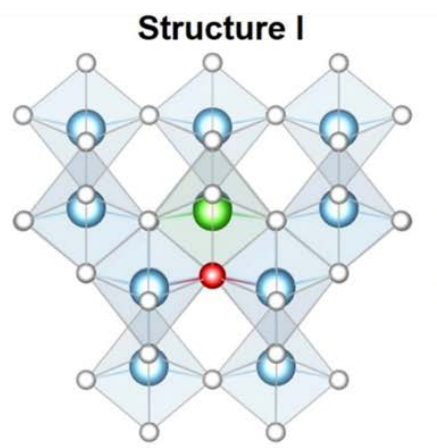

(b)

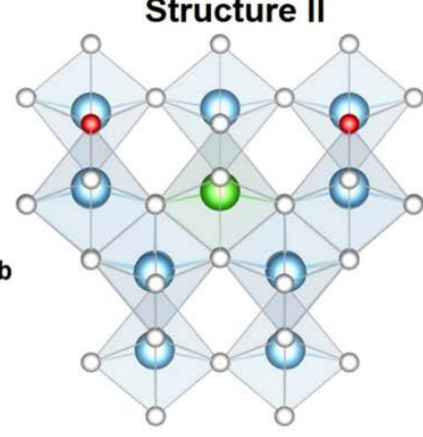

(c)

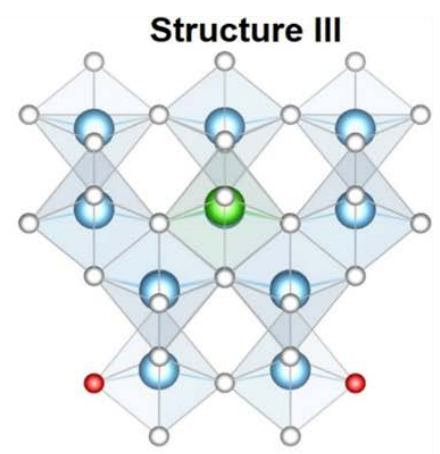

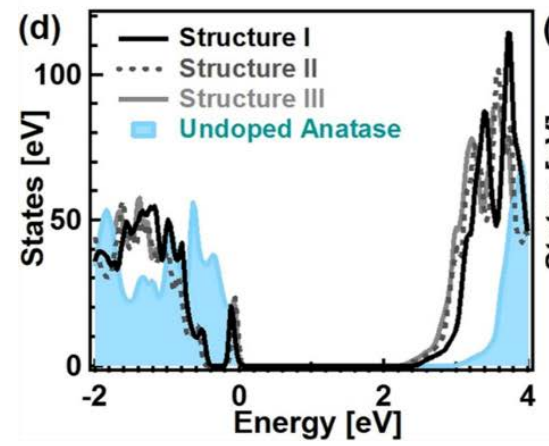
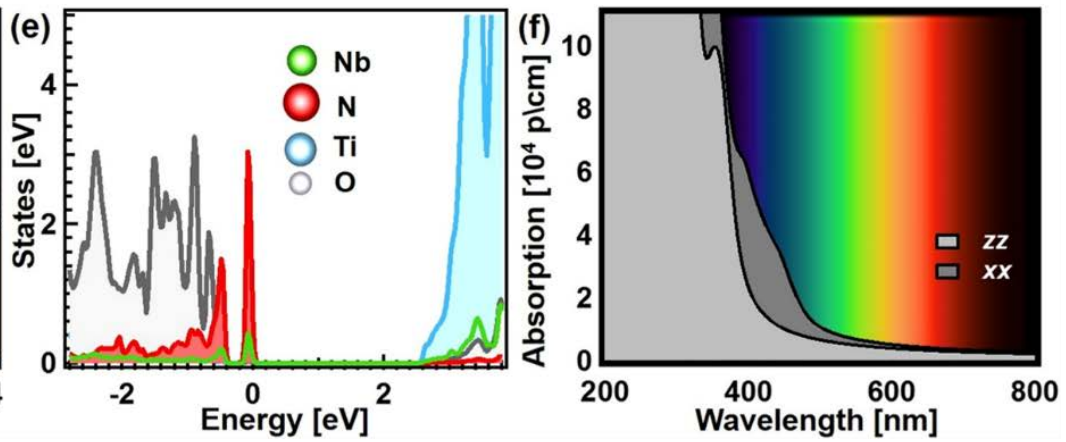

Figure 3. (a-c) Candidate defect-pair chemical structures (I-III) considered for DFT calculations of substitutional $\mathrm{Nb}$ and $\mathrm{N}$ in anatase $\mathrm{TiO}_{2}$. The dotted circles indicate the symmetry-equivalent position of the nitrogen positioned in the supercell. (d) The total density of states calculated using the $\mathrm{mBJ}$ potential in the three structures compared to un-doped anatase $\mathrm{TiO}_{2}$, with $\mathrm{E}=0$ corresponding the Fermi energy. (e) The partial density of states for the lowest energy defect-pair structure (I) showing the shallow nitrogen acceptor lever in the gap and the $\mathrm{Nb} 3 \mathrm{~d}$ states high in the conduction band. (f) Calculated optical absorption coefficient from Structure I for two components $(x x, z z)$ of the anisotropic optical tensor, showing absorption into the visible light region (400-700 nm) depicted by the overlaid spectrum.

Table 1. Density functional theory calculations for candidate defect-pair chemical structures (I-III). The total energy has been computed using the GGA-PBE functional, whereas the bandgap values were obtained using the $\mathrm{mBJ}$ potential.

\begin{tabular}{lccc}
\hline Samples & Nb-N distance $[\AA]$ & Energy $[\mathrm{eV}]$ & Bandgap $[\mathrm{eV}]$ \\
\hline Structure I & 1.929 & 0 & 2.43 \\
Structure II & 4.289 & 0.116 & 2.40 \\
Structure III & 6.001 & 0.124 & 2.36 \\
Pure Anatase & - & - & 2.98 \\
\hline
\end{tabular}

